\title{
Damage Analysis of Concrete Structure under Multidirectional Shaped Charge Blasting Using Model Experiment and Ultrasonic Testing
}

\author{
Dunwen Liu, Jianjun Zhang $\mathbb{D}^{D}$, Yu Tang $\mathbb{D}^{D}$, Yinghua Jian, and Caiwu Cai \\ School of Resources and Safety Engineering, Central South University, Changsha 410083, China \\ Correspondence should be addressed to Yu Tang; tangyu9433@163.com
}

Received 17 November 2020; Revised 9 February 2021; Accepted 15 February 2021; Published 23 March 2021

Academic Editor: Claudio Mazzotti

Copyright (c) 2021 Dunwen Liu et al. This is an open access article distributed under the Creative Commons Attribution License, which permits unrestricted use, distribution, and reproduction in any medium, provided the original work is properly cited.

\begin{abstract}
Quantitatively analyzing multidirectional shaped charge blasting energy distribution and improving the rock-fracturing efficiency have been a challenging problem in blasting and civil engineering. In this work, we carry out four groups of concrete model experiments using ultrasonic testing, comparing conventional blasting, and multidirectional shaped charge blasting. Then, the probability and statistics method is used to quantitatively analyze the blasting damage and the energy distribution. The test results show that ultrasonic testing and statistics model can quantitatively evaluate the damage law and energy distribution of blasting. By comparing with conventional blasting method, the multidirectional shaped charge blasting with V-shaped multidirectional shaped energy groove has achieved the effect of energy accumulation. It increases the distribution of energy in the rock crack district, increases the blasting damage range, and improves the rock-fracturing efficiency of blasting. The V-shaped multidirectional shaped energy groove can be used as a new approach for rock fracturing in similar projects.
\end{abstract}

\section{Introduction}

As a primary method of rock mass excavation, the drilling and blasting method has been widely used in geotechnical engineering, such as tunnel engineering, slope engineering, and foundation pit engineering. However, with the strict requirements of safety, quality, and cost control in engineering construction, additional requirements on the utilization of blasting energy have been proposed. How to properly distribute blasting energy between rock fracturing and rock crushing in order to enhance the utilization of the blasting energy is an urgent issue to be resolved in blasting engineering.

The research on the control of blasting energy in various countries mainly focuses on the shaped charge. This is because the consequent jet flow can be used to realize the distribution of the blasting energy. As such, it is widely applied in both military and civil explosive fields. Most of the earlier studies on the shaped charge blasting focus on the mechanism and the action characteristics of the shaped charge jet, while those on the application of the shaped charge blasting in rock fracturing are limited. In the 1970s, the shaped charge blasting was applied in geotechnical engineering. A groove-type reflector was used to conduct the fluid-solid-decoupled charging, and the blasting energy induced a shaped hydraulic jet flow, which solves the problem of overbreak in the tunnel excavation process. Subsequently, Fourney et al. developed a method to obtain a directional crack formation in the hole of an axial slit tube charge [1]. A Swedish engineer, Bjarnholt et al., conducted the engineering blasting with a linear-shaped energy-concentrated charge [2]. He et al. developed the new technology of bidirectional shaped tensile blasting, and the corresponding shaped apparatus which could perform tension fracture by forming on a blasting mass along the desired direction [3]. Yang et al. conducted the contrast test of the shaped charge blasting, optimized the blasting parameters, and achieved application effects on the rapid excavation in hard-rock tunnels $[4,5]$. Meng et al. used the energy-shaped charge blasting technology to directionally break the hard 
roof and release the mining pressure. After the initiation, blasting-induced fractures mainly propagated in a shaped charge jet direction between the adjacent boreholes [6]. Tian et al. used the action mechanism of empty-hole directional blasting to study the influence of charge hole spacing on crack propagation [7]. Song et al. established five finite element models of cumulative blasting to study the effect of decoupling coefficient on cumulative blasting to improve coal seam permeability [8]. In the abovementioned studies, a good application effect was achieved by the bidirectional linear-shaped charge blasting on smooth blasting. However, in the construction of geotechnical engineering, the issue of larger rock block after blasting exists, and the energy utilization is limited. Therefore, as a new idea of rock breaking, the multidirectional shaped charge blasting was proposed. In order to study the crack propagation mechanism of multidirectional shaped charge blasting, Luo and Shen attached four wedge-shaped liners symmetrically to the cast charge and achieved good application effects in which the orientation fracture blasting with a shaped charge can be used in fracture controlled blasting [9]. Wang used an irregular shape cartridge and revealed the rock breaking mechanism of directional fracture blast [10]. The earlier studies on the multidirectional shaped charge blasting mainly used field tests and numerical simulations. Owing to the complex conditions in the engineering blasting field, only a qualitative description is available for the utilization of blasting energy and the distribution rule of blasting energy. Hence, the quantitative description of the multidirectional shaped charge blasting energy and the corresponding mechanisms of the rock blasting need further investigation.

Therefore, in this study, based on the blasting test of an analogical concrete model and the characteristics of rock blasting, the test data of ultrasonic wave speed have been used to quantitatively describe the energy utilization of the shaped charge blasting through the approaches of theoretical analysis and mathematical statistics. It can be used as a reference for the application of multidirectional shaped charge blasting technology in blasting engineering.

\section{Theory of Multidirectional Shaped Charge Blasting}

2.1. Characteristics of Blasting Action. According to the conventional blasting theories, the rock-fracturing process by blasting is a joint action of detonation gas pressure and stress wave. When the blasting cartridge is embedded in a rock mass and under a constraint condition, enlarged cavity, smash district, crack district, and vibration district will be formed after blasting [11], as shown in Figure 1.

From Figure 1 above, it can be seen that, after blasting, the pore wall of the charged district is subjected to high temperature, high pressure, and shock waves, resulting in an enlarged cavity formed by forced squeezing. The ultrahigh shock pressure induces shock waves in the rock mass and causes oversmashing of the rock surrounding the charge. This results in the formation of the smash district, the range of which is usually two to three times the charge radius, which consumes a lot of the blasting energy. Then, some

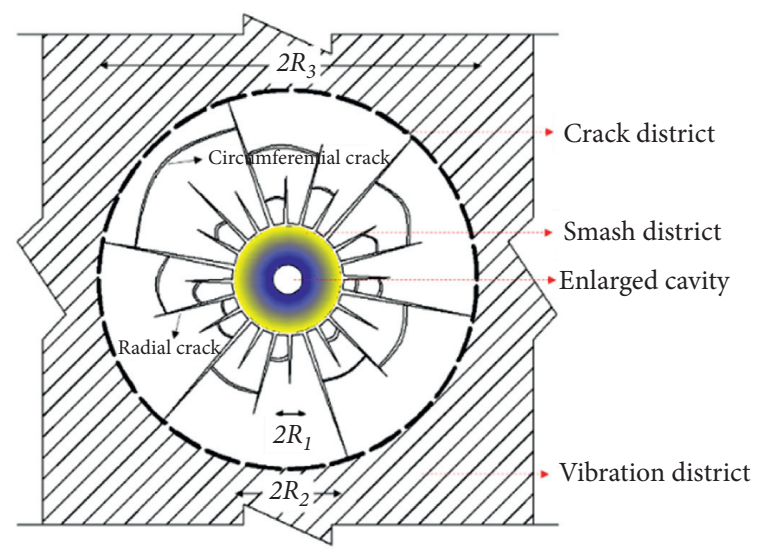

FIgURE 1: Division of rock blasting action.

radial and circumferential fractures are produced outside the smash district and form the crack district, the range of which determines the utilization of blasting energy. Outside the crack district is the vibration district, where the stress wave attenuates to sinusoidal wave, causing merely elastic vibration to the rock mass, and the energy of this district makes up $3 \%-15 \%$ of the total blasting energy $[12,13]$.

\subsection{Rock-Fracturing Characteristics of Multidirectional} Shaped Charge Blasting. Regarding the shaped charge blasting, the cartridge of a given specification is installed into a shaped charge apparatus, and the groove-type structure with an energy accumulation function is machined in the apparatus in different directions along a given angle. After the blasting of the conventional spherical or cylindrical cartridge, the detonation products of high temperature and high pressure spread irregularly along the radial direction of the blast hole. Furthermore, the shaped charge blasting applies an uneven blasting load on the pore wall. The shaped charge apparatus is utilized to change the pressure distribution on the pore wall caused by the detonation products so as to first produce cracks at the predetermined locations on the pore wall [14]. In this study, the V-type groove of 5direction shaped charge blasting in the tests has been used as the example. Figure 2 shows the multidirectional shaped charge jet penetrating rock mass.

From Figure 2, it can be seen that, under the effect of the shaped charge apparatus, after blasting, the explosive shock wave is first unloaded in the direction of the shaped groove. Additionally, the shaped jet flow caused by the blast around the shaped charge groove acts on the pore wall to form a preliminary radial crack [15]. Owing to the rapid action duration, the energy caused by the blasting has not been fully consumed; about $50 \%$ of the energy continues to exert pressure on the cracked rock in the form of detonation gas. Under the effect of "air wedge" of detonation gas, the crack develops further, which separates the affected region from the parent rock, and can eventually cause the rock in the region to be thrown away.

The shaped charge blasting fully uses the characteristics of the "resistive to compression and vulnerable to tension" of rock, which causes the formation of tangential tensile stress 


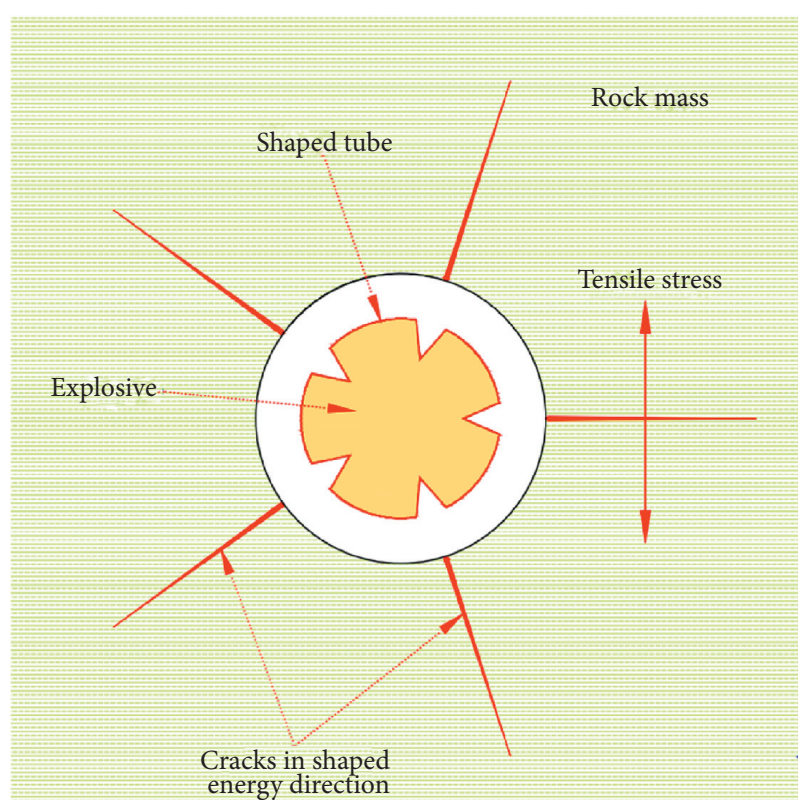

FIGURE 2: Multidirectional shaped charge jet penetrating rock mass.

that is vertical to the shaped charge direction through the coupling effect of the shaped charge groove and the explosive charge $[16,17]$. The tangential tensile stress causes the initial crack on the pore wall of the blast hole. When the radial tensile stress caused by the explosive stress wave is applied on the rock between two adjacent principal cracks, the rock can be pulled apart to form a circumferential crack, and the cracks can cut through one another, thereby causing rock fractures [18]. The remaining gas pressure throws out the rock mass, making it separate from the parent rock, and converting the detonation pressure to a tensile effect on the surrounding rock to the maximum extent.

\section{Model Test}

3.1. Physical Model. To quantitatively describe the energy distribution of multidirectional shaped charge blasting and the mechanism of a blasting action, in the model tests of this study, C50 concrete has been used to construct the physical model. The tests have been performed by comparing two groups of single-hole and dual-hole blasting tests with two models in each group, and in total, there are four cuboid models of $1 \mathrm{~m} \times 1 \mathrm{~m} \times 0.9 \mathrm{~m}$. The concrete mix proportions for the models are shown in Table 1. In concrete models, the $40 \mathrm{~mm}$ diameter and $400 \mathrm{~mm}$ depth blast holes are prefabricated in the models. The concrete model test is shown in Figure 3.

No. 2 rock emulsion explosives and the ordinary millisecond electric detonators have been used in the tests. The detonation velocity of No. 2 rock emulsion explosives is more than $4600 \mathrm{~m} / \mathrm{s}$. The explosive load is $30 \mathrm{~g}$ for the singlehole tests. For the dual-hole tests, the explosive load is $20 \mathrm{~g}$ for each blast hole. Therefore, the total explosive load is $40 \mathrm{~g}$. Owing to limited explosive load, the length-diameter ratio of the charge cartridge is smaller than six so that the tests can be considered as spherical cartridge blasting tests.

3.2. Test Plan. Ultrasonic wave speed reflects the capability of penetration and reflection of stress wave in concrete medium, and the fissures (or cracks) in the medium play the most important role in influencing the propagation of ultrasonic wave in concrete. Under the conditions of fissures or cracks, the propagation path of ultrasonic waves is extended, and the speed of ultrasonic waves reduces accordingly. The degrees of reduction in the ultrasonic wave speed are closely associated with the number and the width of cracks. Therefore, in the model tests of this study, the speed test on the longitudinal wave has been conducted on the concrete specimens before and after the blasting. The damage degree and the distribution law of concrete are determined by comparing the characteristics of the variation of the ultrasonic wave speed before and after the blasting. The ultrasonic testing system of concrete is shown in Figure 4.

In Figure 4, the ultrasonic testing system is composed of transmitting transducer, receiving transducer, ultrasonic detector, and computer. The measuring points are arranged on both sides of the concrete test block parallel to the blast hole direction, and the layout of measuring points is shown in Figure 5. Grease is used as a coupling agent, which is evenly applied on the measuring points of the concrete model to ensure there is sufficient coupling between the transducer and the wall at the measurement point of concrete. The transmitting transducer is started by the software inside the ultrasonic detector, and the ultrasonic wave is transmitted from the transmitting transducer to the receiving transducer through the concrete block. Then the receiving transducer transmits the acoustic signal back to the ultrasonic detector to complete the acquisition of a measuring point. During the measurements, the data are recorded when the ultrasonic wave waveform is stable.

In the range of $30 \mathrm{~cm}-85 \mathrm{~cm}$ along the height direction of the model, there are 11 layers, and in each layer, the measurement points are arranged every $5 \mathrm{~cm}$. In the range of $10 \mathrm{~cm}-90 \mathrm{~cm}$ along the width direction, there are 16 measurement points arranged in each layer of the height direction. There is a total of 176 measurement points.

\subsection{Parameters of Multidirectional Shaped Charge Pipe.}

The material of the shaped charge pipe, the shape of the shaped charge groove, and the number of shaped charge grooves determine the effect of rock fracturing by the shaped charge blasting. The parameter selection criteria of the shaped charge pipe are listed as follows.

\subsubsection{Material of the Shaped Charge Pipe. A shaped charge} pipe usually uses material that is of small compressibility, high density, and excellent plasticity and ductility. Further, it uses material that suffers no gasification in the formation of shaped charge jet flow [19]. Based on these features, together with the difficulty in manufacturing a shaped charge pipe, 
TABLe 1: The concrete mix proportions.

\begin{tabular}{lccccccc}
\hline \multicolumn{7}{c}{$1 \mathrm{~m}^{3}$ concrete material consumption $(\mathrm{kg})$} \\
\hline Grade strength & Cement & Fly ash & Slag & Fine aggregate & Coarse aggregate & Water & Water reducing agent \\
\hline C50 & 429 & 47 & 47 & 814 & 956 & 157 & 8.9 \\
\hline
\end{tabular}

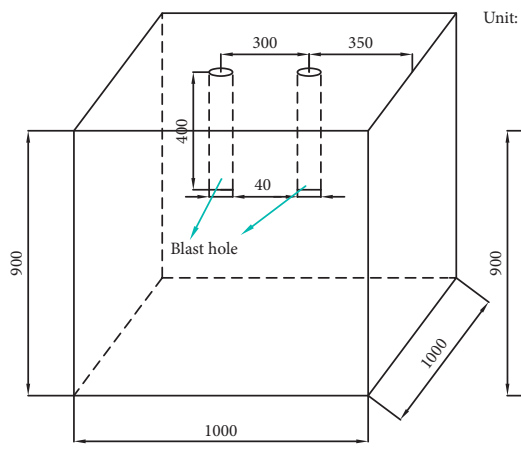

(a)

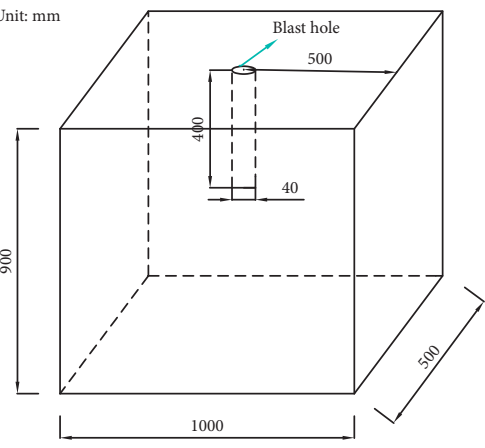

)

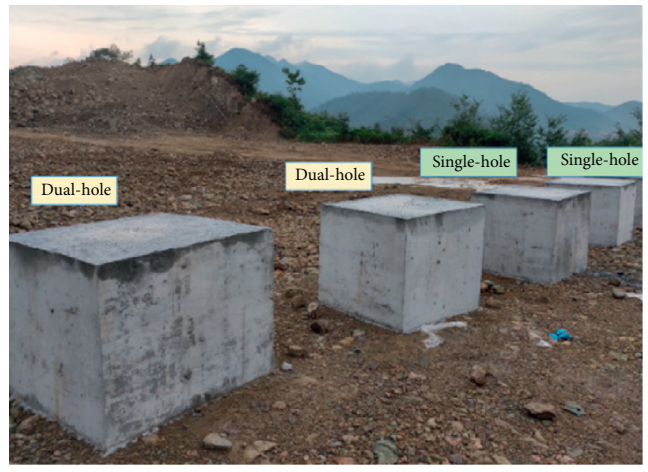

(b)

FIgure 3: Concrete model test: (a) model size; (b) field model tests.

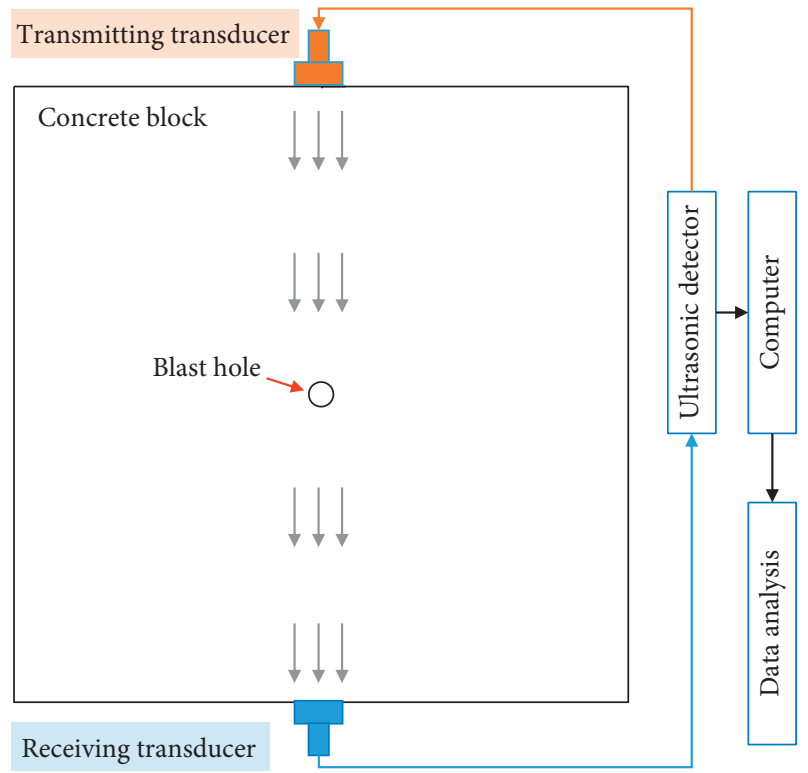

Figure 4: Ultrasonic testing system.

red steel paper has been innovatively selected as the material for the shaped charge pipe. Red steel paper is a processed product with high hardness which is close to that of aluminum. So, the physical properties can meet the requirements as stated above.

3.3.2. Shape of the Shaped Charge Groove. A V-type shaped charge groove has been used. The opening angle of the $\mathrm{V}$ determines the capabilities of penetration and cracking of shaped charge jet flow. It has been demonstrated by the earlier studies that when the angle of the shaped charge groove is in the range of $30^{\circ}-70^{\circ}$, the generated shaped charge jet flow has

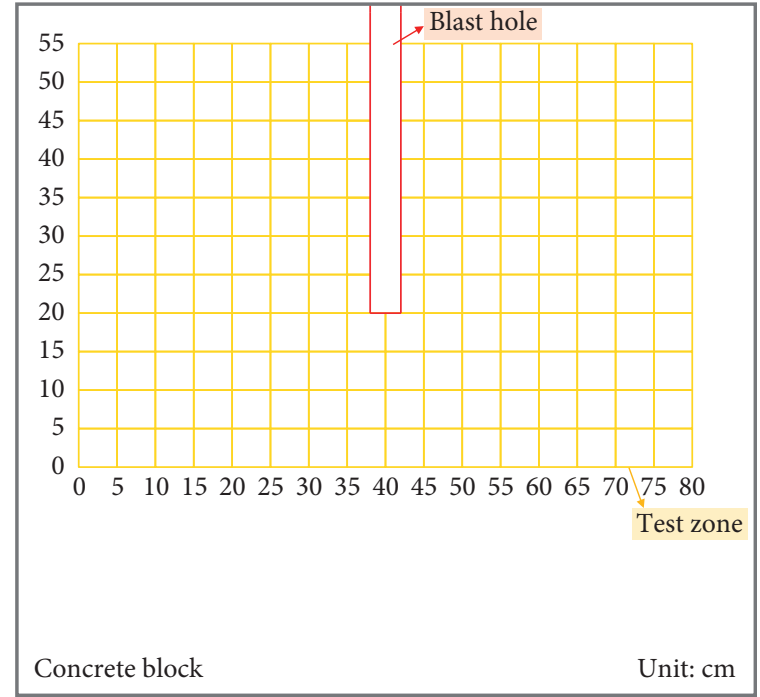

FIGURE 5: Layout of measuring points.

sufficient mass and velocity to cause cracking and piercing. Within this range of angle, as the angle increases, the penetration capability of the shaped charge jet flow gradually decreases while the jet flow mass gradually increases [20-22]. In the early stage of the tests, via numerical simulations of the shaped charge blasting, together with the relevant engineering cases, it has been derived that the optimal angle of the V-type shaped charge groove is $60^{\circ}-75^{\circ}$. The designed angle in the tests of this study is $60^{\circ}$.

3.3.3. The Number of Shaped Grooves. With a certain amount of explosive charge, the energy of a shaped charge jet flow decreases as the number of shaped charge grooves 
increases, and the number of shaped charge grooves has a significant influence on the crack development. It has been demonstrated by the earlier studies that when the number of shaped grooves is $4 \sim 6$, it can both ensure there is sufficient energy in the jet flow and cause the ideal number of cracks. The tests in this study focus on the damage law of the radially fracturing rock mass, which has high requirements on the depth and number of cracks. Hence, five shaped charge grooves have been designed. Based on the discussions of the above factors, the multidirectional shaped charge pipes in the tests are made of high-strength red steel paper. The thickness, density, and diameter of the pipes are $0.12 \mathrm{~cm}$, $1.4 \mathrm{~g} / \mathrm{cm}^{3}$, and $3.5 \mathrm{~cm}$, respectively. The number of shaped charge grooves is 5 , i.e., each shaped pipe with $5 \mathrm{~V}$-type grooves evenly distributed on the pipe wall. The radial length and angle of the pipes are $0.8 \mathrm{~cm}$ and $60^{\circ}$, respectively. According to the dimensions of the physical model, the length of shaped charge pipe is $10 \mathrm{~cm}$, and that of the charge is $5 \mathrm{~cm}$. The multidirectional shaped charge pipe and its cross section are shown in Figure 6.

\subsection{Test Procedure.}

(1) An end of the multidirectional shaped charge pipe is flatly sealed with round red steel paper by adhesive tape, and the emulsion explosive is squeezed into the pipe from the other end

(2) The electronic detonator is inserted into the center of a charge cartridge and the top of detonator is aligned with the top end of the cartridge

(3) The stemming made by mixing sand, clay, and water is used to fill the remaining space in the top region of the shaped pipe and the end is flatly sealed by adhesive tape

(4) The charge tube is loaded to the bottom of a blast hole by a gun barrel, and the remaining space of the blast hole is filled with stemming

(5) The leading wires of the detonator are connected with an exploder and the detonator is actuated beyond a safe distance

(6) After no-dud is confirmed, wait for 5 min and then enter the blasting field and measure the cracks, residual hole, and ultrasonic wave data using the tools such as the measuring instrument and ultrasonoscope

(7) The design explosive loads are adjusted according to the tests of each group, and steps $1 \sim 6$ are repeated

\section{Results and Discussion}

\subsection{Blasting Effects}

4.1.1. Single-Hole Blasting. The macroscopic damage and crack propagation of the concrete models after blasting by the conventional charging and the multidirectional shaped charging are exhibited in Figure 7.

It can be seen that, after the conventional blasting, along the direction of minimum burden on the four free surfaces of the concrete model surfaces, four fissures or cracks are observed. One group of crack penetrates the center of the blast hole and extends to the bottom face of the model, with a width of $6 \mathrm{~mm}$, while the other group can be observed on the top and the side surfaces of the model, with a width of 1-2 $\mathrm{mm}$, as marked at the corresponding locations in Figure 7. No crushing is found when the concrete specimen is blasted by this approach. Thus, the specimen can only be removed by a secondary blasting or chipping-away by a large machinery, demonstrating that this approach leads to only internal fracture inside the concrete. For the concrete models subjected to the multidirectional shaped charge blasting, three fracture penetrating fissures whose maximum width is $12 \mathrm{~cm}$ are produced along the direction of minimum burden from the center of the blast hole, the depth of which reaches the bottom of the model, and there is one $8 \mathrm{~mm}$ wide penetrating fissure, indicating that the destruction is significant.

The test results indicate that, with the same explosive load, both blasting approaches lead to four fissures on the model surface but the degrees of the fissure development are significantly different. The fissures and the degree of destruction caused by the shaped charge blasting are far larger than those by the conventional blasting. The load of the conventional blasting acts evenly on the concrete around the explosive resulting in random cracks. The propagation direction of the principal crack is influenced by the distribution of free surfaces of concrete, which is approximately consistent with the direction of minimum burden. The multidirectional shaped charge blasting alters the action direction of the stress wave and detonation gas, which concentrates the energy in the direction of the shaped charge grooves so that the damage of the surrounding concrete in this direction is larger and the damage depth of crack is longer. However, since the explosive loads of the tests in this study are relatively limited and the shaped charge grain is too short for practical use, the shaped charge blasting effect is therefore weaker than the effect of the free surfaces on the blasting effect. Thus, the macroscopic damage of the concrete model is expressed as the fracture runs along the direction of the minimum burden, with enhanced degree of damage.

4.1.2. Dual-Hole Blasting. The concrete damage patterns caused by the dual-hole blasting of the two charging approaches are shown in Figure 8.

Both charging approaches cause the model to explode into two parts along the direction of the connecting line of the blast holes, among which the conventional blasting pushes half of the concrete over and the multidirectional shaped charge pushes both parts of the concrete to corresponding sides. It is clear that the stronger impact on the model is caused by the multidirectional shaped charge blasting. Thus, it can overcome the constraint of the concrete cohesion better. The cracks around the blast hole are shown in Figure 9.

It can be seen from Figure 9(a) above, the fractured surface of the model caused by the conventional blasting is 


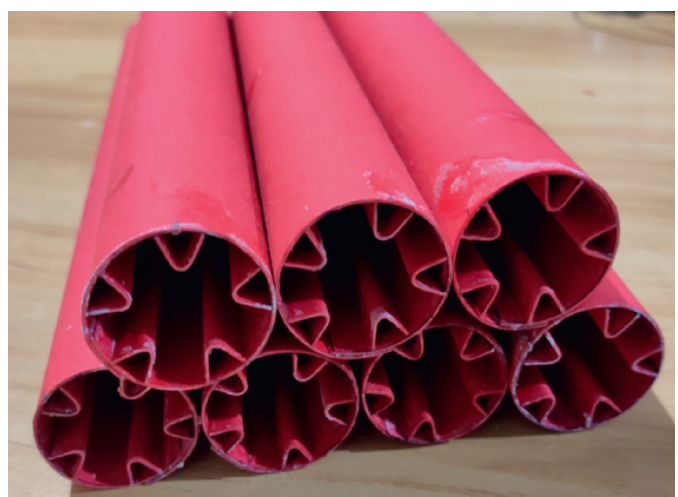

(a)

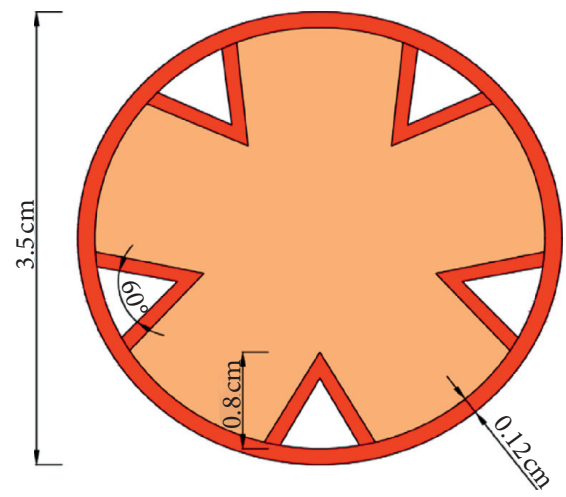

(b)

FIGURE 6: Multidirectional shaped charge pipe: (a) product; (b) section size.

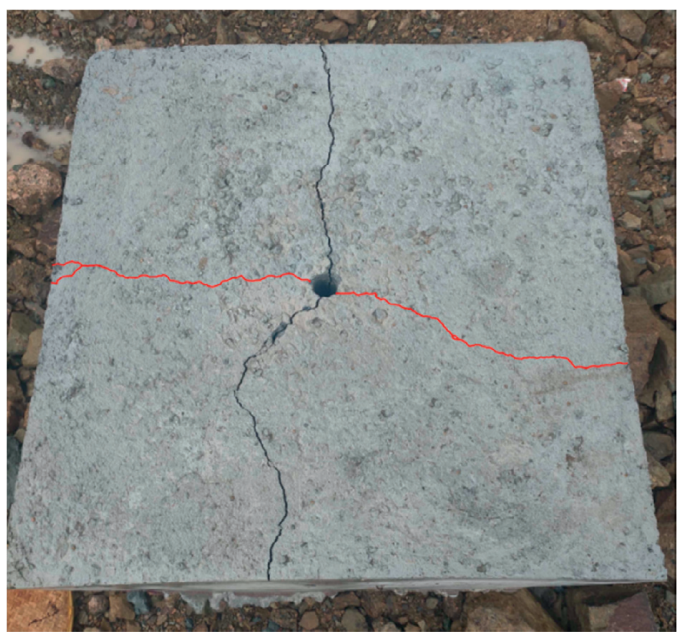

(a)

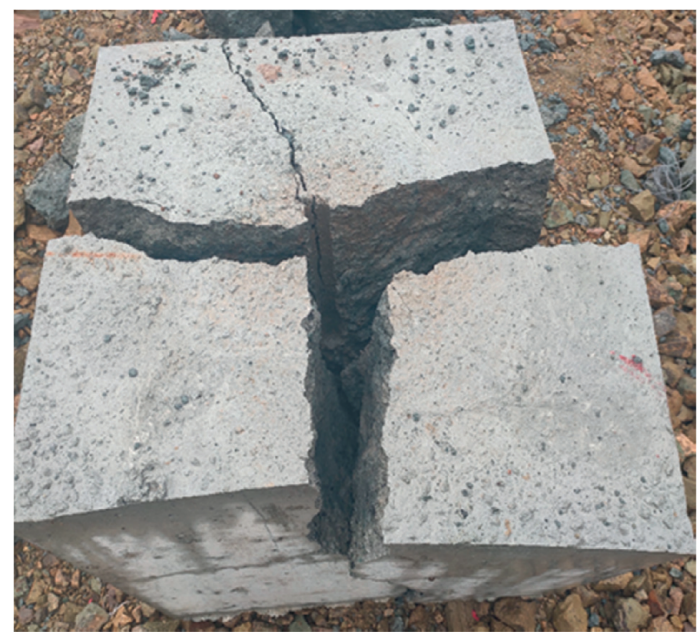

(b)

FIGURE 7: Damage and crack propagation of single-hole blasting: (a) conventional blasting; (b) multidirectional shaped charge blasting.

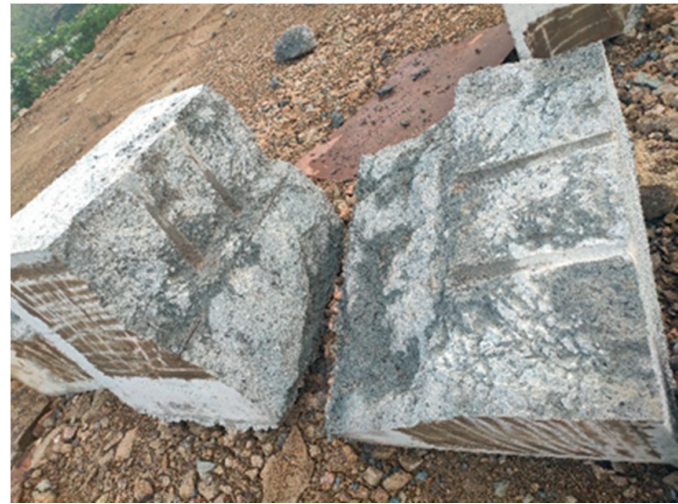

(a)

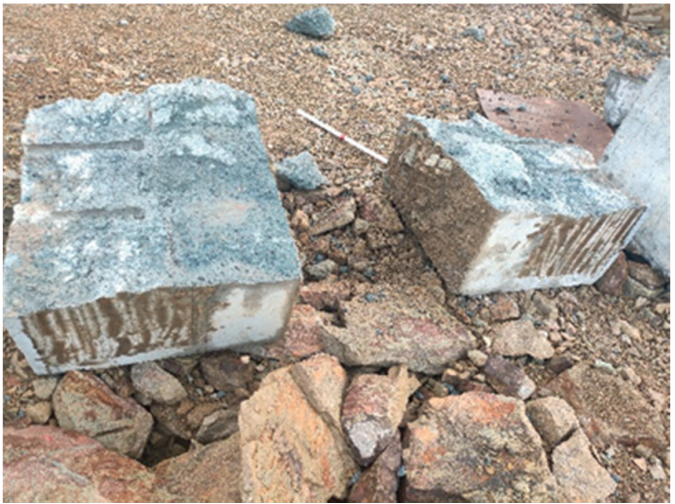

(b)

FIGURE 8: Damage and crack propagation of dual-hole blasting: (a) conventional blasting; (b) multidirectional shaped charge blasting. 


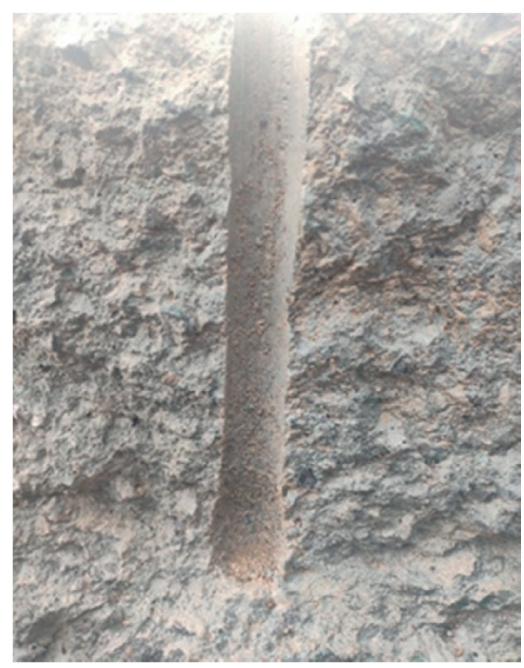

(a)

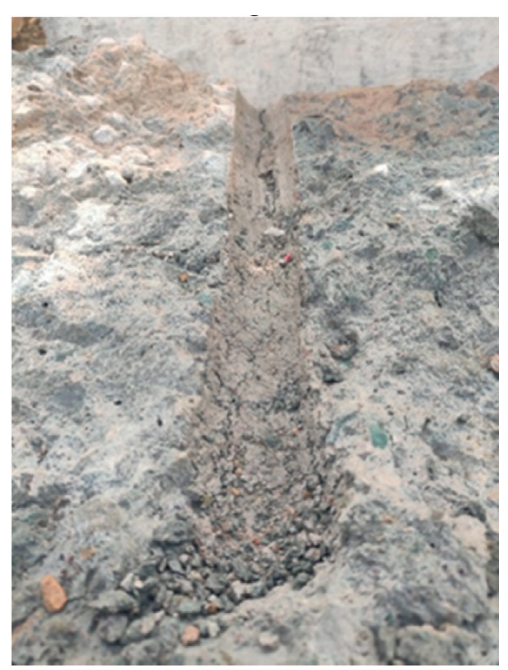

(b)

FIGURE 9: Cracks around the blast hole after blasting: (a) conventional blasting; (b) multidirectional shaped charge blasting.

relatively smooth. An enlarged cavity appears in the charging space in the bottom of the blast hole, with a diameter of $6 \mathrm{~cm}$. No distinct damage cracks are observed inside the model. The fractured surface of the model caused by the multidirectional shaped charge blasting is not smooth, as shown in Figure 9(b). The bottom space of the blast hole is crushed, with an enlarged cavity appearing, and the diameter of the cavity is $7 \mathrm{~cm}$. Fine cracks spread all over the axial direction of the blast hole and they penetrate one another. Therefore, the multidirectional shaped charge blasting causes greater damage to the internal structures of the concrete.

4.2. Analysis of Ultrasonic Test Results. The differences in the test results of both single-hole blasting tests are considerable. Further, the model for the multidirectional shaped charge blasting is severely damaged, which makes the postblasting test of the ultrasonic wave difficult. Therefore, in this study, the analysis has been carried out with the ultrasonic wave test results of the before and after dual-hole blasting.

The measured value of ultrasonic velocity in the concrete around the blast hole is around $4.21 \mathrm{~km} / \mathrm{s}$ before blasting, and those at the other measurement points are around $4.27 \mathrm{~km} / \mathrm{s}$. So, the model is regarded as an approximate continuum uniform medium. After blasting, the ultrasonic velocity in the concrete model decreases, and it gradually decreases as the distance between the measurement point and the blast hole increases. According to the specifications, when the decrease rate of ultrasonic velocity $\eta<10 \%$, it is considered that blasting has little or no influence on the rock mass. If $10 \% \leq \eta \leq 15 \%$, the influence of blasting on a rock mass is slight. If $\eta>15 \%$, blasting has an influence on a rock mass. To directly reflect the variation rule of ultrasonic wave speed at the inner measurement points of concrete after blasting, the ultrasonic velocity before blasting is taken as the initial value to calculate the decrease rate of the ultrasonic wave speed $\eta$ after blasting. Further, the decrease rate of the ultrasonic wave speed at the measurement point is represented by the chromatographic contour, as shown in Figure 10. In the figure, the shaded area denotes the measurement region around the fractured surface which is severely damaged, which makes acoustic detection impossible.

It can be seen from Figure 10 above that, under the action of blasting, as the explosive is placed at the bottom of the blast hole, the concrete model has different degrees of damage. With the explosive as the center, the damage degree gradually weakens from the explosive center to the outside. Therefore, the ultrasonic wave speed isolines present inhomogeneous distribution. The details are as follows.

After blasting, regarding the measurement points at the bottom and on the wall of the charging hole, the degree of decrease of the ultrasonic wave speed is significantly larger than those in other areas. Further, the degree of the ultrasonic wave speed decreases gradually as the distance from the charging region increases. After multidirectional shaped charge blasting, in the region range with the width of $20-30 \mathrm{~cm}$ and the length of $32-52 \mathrm{~cm}$, the degree of decrease of the ultrasonic wave speed is more than $74 \%$. The degree of decrease of the ultrasonic wave speed at the two sides of the blast hole is generally larger than that near the free surface. The decreasing rates of the ultrasonic wave speed along the axis of the blast hole and in the direction perpendicular to the blast hole in the charging region tend to reduce. However, that on the free surface along the axial direction of the blast hole is larger than that on the free surface in the direction perpendicular to the blast hole. The rate of decrease of the ultrasonic wave speed in the former measurable region is $28 \%$ at maximum while that in the latter is generally $19 \%$. After the conventional blasting, the relatively large decrease in the ultrasonic wave speed 


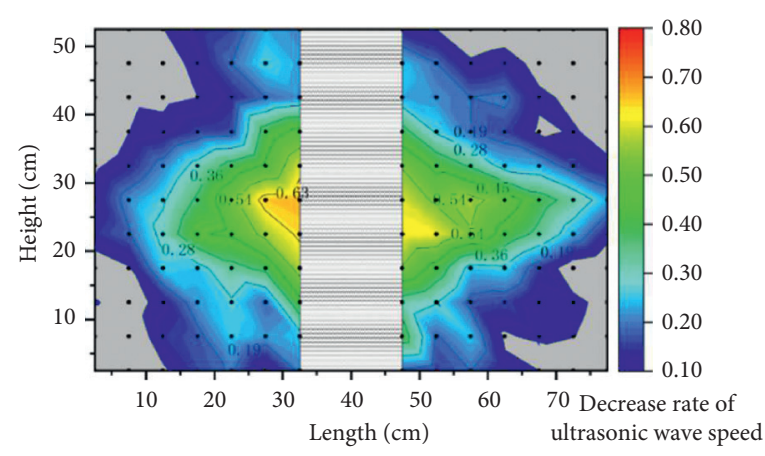

(a)

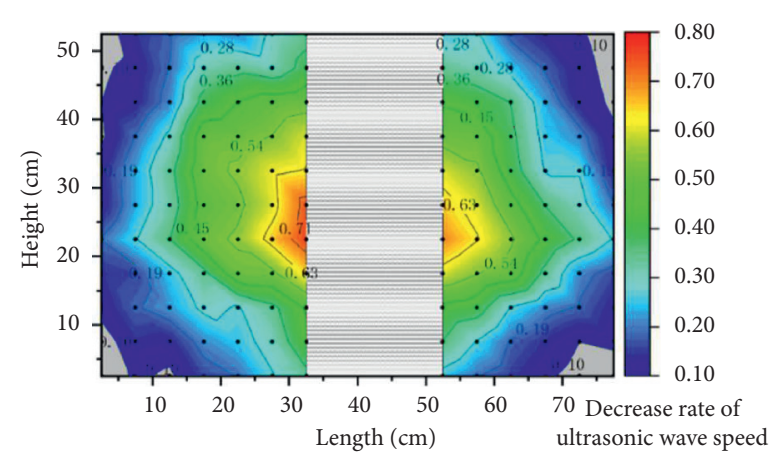

(b)

FIGURE 10: Reduced rate of sonic velocity after blasting: (a) conventional blasting; (b) multidirectional shaped charge blasting.

occurs at the bottom of the blast hole, which reaches more than $63 \%$. It decreases towards the free surfaces of both sides of the blast hole. The region is limited and the distribution is irregular. At the height of $20 \mathrm{~cm}$ from the bottom of the blast hole, the rate of decrease of the ultrasonic wave reduces to $19 \%$ while the reductions are less in other regions. The degree of concrete damage has the following relationship with the decrease rate of ultrasonic speed:

$$
D=1-\frac{E}{E_{0}}=1-\left(\frac{v}{v_{0}}\right)^{2}=1-(1-\eta)^{2},
$$

where $D$ is the damage degree, $v$ is the measured ultrasonic wave speed, $\mathrm{km} / \mathrm{s}, v_{0}$ is the initial ultrasonic wave speed of concrete, $\mathrm{km} / \mathrm{s}$, and $\eta$ is the decrease rate of ultrasonic wave speed.

The damage degree of concrete has the following relationship with the rate of decrease of ultrasonic wave speed. Based on the ultrasonic wave speed of the concrete model before blasting, the ultrasonic wave speed after blasting at each measurement point is substituted into (1) to obtain the damage degree $D$ of concrete under different charging structures after blasting. The chromatographic contours of the damage degree are shown in Figure 11.

From the chromatographic contours of the damage, the following can be seen:

(1) After the conventional blasting, for $D>0.19$, the damage radius of the stemmed blast hole and the detonation section of the detonator is around $15 \mathrm{~cm}$. As the depth of the blast hole increases, the damage radius of the charging section rises to $35 \mathrm{~cm}$ and the damage is extended to $20 \mathrm{~cm}$ below the bottom of the blast hole. The damage degree rapidly decreases as the distance from the charging center increases. The damage contour varies along the center of charging towards the free surface, and a part of undamaged area exists in the measurement region farthest away from the blast source. This is an indication that this region is only subjected to the disturbance of the explosive elastic wave, which is consistent with the feature that no distinct crack exists on the model surface after real blasting as shown in Figure 7(a).

(2) After the blasting by the multidirectional shaped charging structure, for $D>0.19$, the concrete in the blast hole region is completely destroyed, and the impact scope extends to the free surfaces. The damage degrees of both sides of the blast hole are basically consistent, and the ratio of radius to depth of the failure zone is around $1: 1.1$. The damage contour shows a pattern of approximate uniform scattering to the surrounding. Both the degree and the range of damage are considerably larger than those by the conventional blasting.

To quantitatively analyze the damage law and energy distribution characteristics of concrete under different blasting approaches, the probability statistical analysis has been performed for the damage degrees at all the measurement points. The probability density histogram and its fitting curve are shown in Figure 12.

After the conventional blasting, the largest damage degree of the measurable region of concrete model reaches 0.89 , and the probability density shows attenuation distribution generally. The probability of damage degree of $0.2 \sim 0.5$ is $65.1 \%$, where the damage degree of $0.2 \sim 0.3$ indicates the maximum probability event through the whole interval, the probability of which is $26.6 \%$. In the damage degree of $0.5 \sim 0.6$, the probability drops significantly, and the degree of decrease of distribution probability is $11.9 \%$ as compared with that of the previous interval. In the damage degree of $0.6 \sim 0.9$, the probability density distribution is relatively gentle. The mean distribution probability is $9.5 \%$ for the three small intervals involved and the total distribution probability of this section is only $28.5 \%$, which is consistent with the low proportion of the area of the warmcolor region on the damage contour in Figure 11. The probability distribution of the damage degree of the conventional blasting conforms to the Birnbaum-Saunders distribution. The probability density histogram and its fitting curve of damage degree by multidirectional shaped charge 


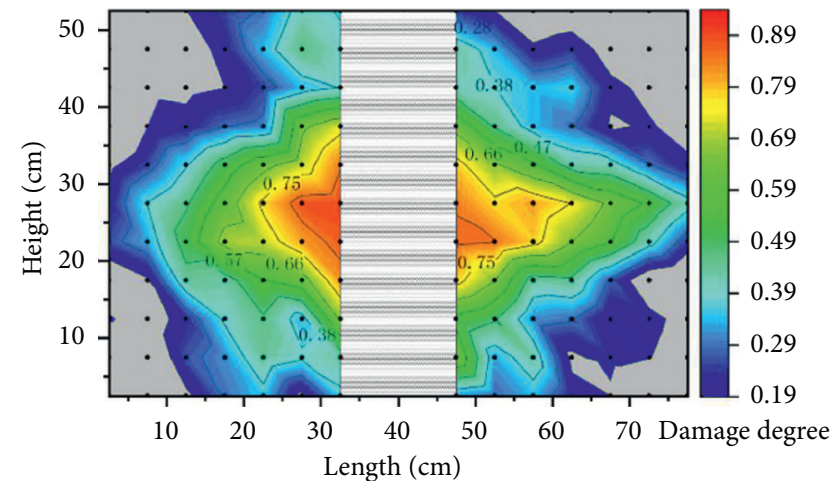

(a)

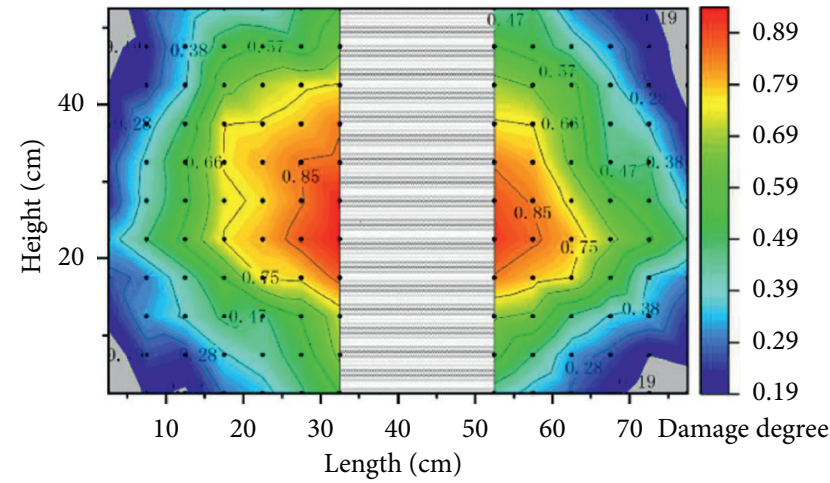

(b)

FIgURE 11: Damage degree after blasting: (a) conventional blasting; (b) multidirectional shaped charge blasting.

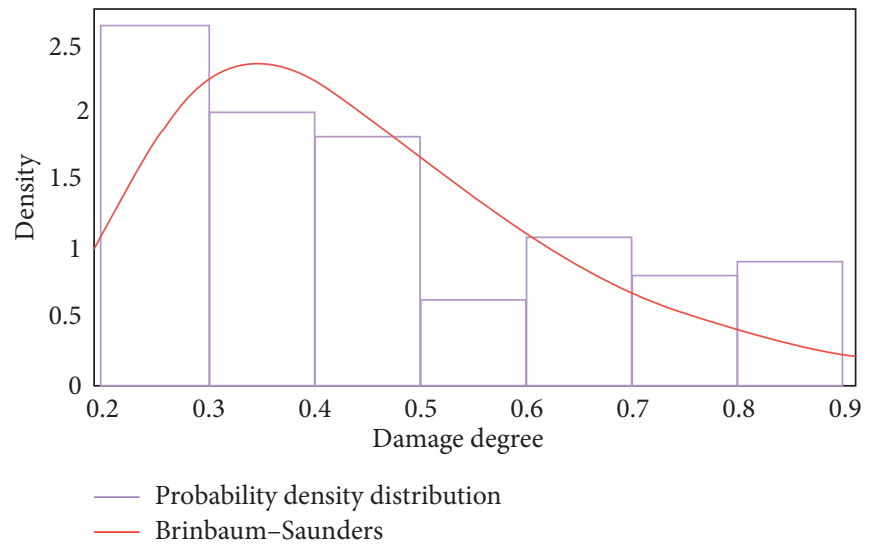

FIgURE 12: The probability density histogram and fitting curve of damage degree by conventional blasting.

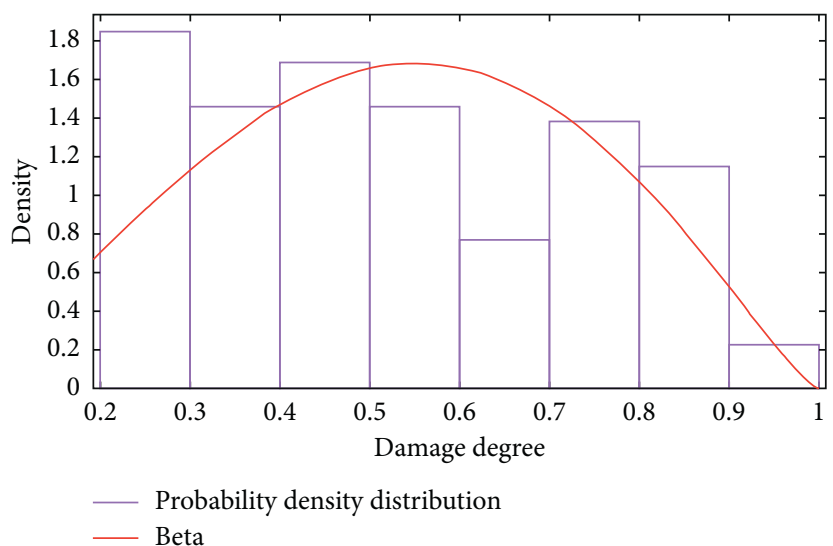

FIGURE 13: The probability density histogram and fitting curve of damage degree by shaped blasting.

blasting are shown in Figure 13.

After the multidirectional shaped charge blasting, the largest damage degree of concrete model is 0.94 , and the probability density distribution is relatively uniform as a whole. The distribution probability is $50 \%$ for the damage degree of $0.2 \sim 0.5$, where the damage degree of $0.2 \sim 0.3$ indicates the event with the largest probability in the whole section, which is $18.5 \%$. In the damage degree of $0.6 \sim 0.9$, comparing with that of the conventional blasting, the distribution probability of the damage degree of the shaped charge blasting has improved to $33 \%$. Particularly in the damage degree of $0.9 \sim 1.0$, the distribution probability is $2.3 \%$, and by comparing the proportion of the 
warm-color region on the damage contour, a consistent conclusion can be drawn. The probability distribution of the damage degree of the shaped charge conforms to the Weibull distribution.

From the probability density distribution of the two charging approaches above, the following can be seen:

(1) The intervals where the distribution probability of the damage degree of concrete is larger the mean distribution probability of $12.5 \%$ include the damage degree of $0.2 \sim 0.6$ and $0.7 \sim 0.8$. Comparing with the conventional blasting, the high probability intervals for the shaped charge blasting are extended and increased along the positive direction of the horizontal axis. This is an indication that the multidirectional shaped charging structure can cause the damage distribution of the model concentrate to a higher damage degree.

(2) With the damage degree of 0.5 as the critical point, the intervals have been divided into low-damage degree and high-damage degree intervals, namely, damage degree $D<0.5$ as low-damage degree and $D \geq 0.5$ as high-damage degree. So, the probabilities of the damage values of the conventional blasting and the multidirectional shaped charge distributed in the high-damage interval are $34.9 \%$ and $49.9 \%$, respectively. The probability of the damage value in the high-damage interval for the multidirectional shaped charge is 1.43 times that of the conventional blasting. This is an indication that the shaped charge blasting can control the mode of distribution so that more energy is concentrated in the shaped charge direction to cause greater damage to a rock mass, while the damage to the area of low-damage zone caused by the blasting is reduced. In the meantime, the shaped charge blasting can effectively reduce the number of random cracks and avoid the blasting energy dissipation caused by uniform force around the blast hole.

\section{Conclusions}

(1) The V-type shaped charge groove designed for the tests in this study can make the blasting energy concentrate in the form of a shaped charge jet flow on the pore wall in the direction of the shaped charge groove. The strong penetration of the shaped jet flow ensures the initiation and the propagation of the principal radial cracks of the pore wall in the direction of the shaped charge groove, thereby reducing the peak strain significantly in the nonshaped directions of the pore wall, effectively avoiding the dissipation of the blasting energy of the uniform force of the blasting hole wall, and increasing the blasting damage radius and the damage degree.

(2) The multidirectional shaped charge blasting can increase the range of the blasting damage. The effective damage range with the damage degree above 0.19 is 1.25 times that of the conventional blasting.
(3) The probability density distribution of the damage degree of the conventional blasting conforms to the Birnbaum-Saunders distribution, while that of the multidirectional shaped charge conforms to the Weibull distribution. Comparing with the conventional blasting, the multidirectional shaped charge blasting makes the model damage distribution concentrated towards the direction of the relatively higher damage degree. In particular, the distribution probability of the high-damage zone whose damage degree ranges between 0.6 and 1 is 1.43 times that of the conventional blasting.

\section{Data Availability}

The data used to support the findings of this study are available from the corresponding author upon request.

\section{Conflicts of Interest}

The authors declare that there are no conflicts of interest.

\section{Acknowledgments}

The authors would like to thank Road and Bridge South China Engineering Co. Ltd. for their assistance with conducting the field experiments. The research has been supported by the Initiation Fund for postdoctoral research of Central South University (grant no. 228697).

\section{References}

[1] W. L. Fourney, J. W. Dally, and D. C. Holloway, "Controlled blasting with ligamented charge holders," International Journal of Rock Mechanics and Mining Sciences \& Geomechanics Abstracts, vol. 15, no. 3, pp. 121-129, 1978.

[2] G. Bjarnholt, R. Holmberg, and F. Ouchterlong, "A linear shaped charge system for contour blasting," in Proceedings of 9th Conference on Explosives and Blasting Technique, pp. 350-358, Society of Explosives Engineers, Dallas, TX, USA, January 1983.

[3] M. He, W. Cao, R. Shan, and S. Wang, "New blasting technology-bilateral cumulative tensile explosion," Journal of Rock Mechanics and Geotechnical Engineering, vol. 22, pp. 20472051, 2003.

[4] R. S. Yang, X. T. Gao, G. Q. Liu, X. Li, and D. Y. Hao, "The structure design of slit-charge for directional fracture controlled blasting in stone drifting," Advanced Materials Research, vol. 619, pp. 388-392, 2012.

[5] R. Yang, Z. Zhang, L. Yang, and Y. Guo, "Cumulative blasting experiment study of slotted cartridge based on hard-rock rapid driving technology," Journal of Rock Mechanics and Geotechnical Engineering, vol. 32, pp. 317-323, 2013.

[6] N. Meng, Y. Chen, J. Bai, X. Wang, W. Wu, and B. Wu, "Numerical simulation of directional fracturing by shaped charge blasting," Energy Science \& Engineering, vol. 8, no. 5, pp. 1824-1839, 2020.

[7] G. Tian, J. Dai, Y. Wu, X. Xiong, and Z. Chen, "Influence of charge hole spacing on the crack propagation behavior under the effect of empty-hole directional blasting," Advances in Civil Engineering, vol. 2020, Article ID 8860816, 10 pages, 2020. 
[8] Y. Song, X. Li, D. Guo, and B. Shi, "Study on the decoupled charge effect in deep-hole cumulative blasting of coal seam," Advances in Civil Engineering, vol. 2019, Article ID 8486198, 9 pages, 2019.

[9] Y. Luo and Z. Shen, "Study on orientation fracture blasting with shaped charge in rock," Journal of University of Science and Technology Beijing, Mineral, Metallurgy, Material, vol. 13, no. 3, pp. 193-198, 2006.

[10] Y. Wang, "The rock-breaking mechanism of directional fracture blast using an irregular shape cartridge and its field application," Journal of Testing and Evaluation, vol. 47, no. 2, Article ID 20160496, 2019.

[11] N. I. Aleksandrova and E. N. Sher, "Modeling the failure of rock blocks by blasting a cylindrical charge," Journal of Mining Science, vol. 42, no. 1, pp. 27-34, 2006.

[12] H. Tang, Y. Zhou, and Y. Liao, "Damage zone of surrounding rock of underground engineering under construction blasting," Shock and Vibration, vol. 34, pp. 202-206, 2015.

[13] R. Yang, C. Xiao, and Y. Li, "A fractal study on blasting damage of an eccentric decouple charge structure," Shock and Vibration, vol. 39, pp. 134-139, 2020.

[14] L. Liu, Z. Liu, K. Gao, Y. Ma, Z. Li, and L. Guo, "Experimental study and application of directional focused energy blasting in deep boreholes," Journal of Rock Mechanics and Geotechnical Engineering, vol. 33, pp. 2490-2496, 2014.

[15] F. Wang, S. Tu, Y. Yuan, Y. Feng, F. Chen, and H. Tu, "Deephole pre-split blasting mechanism and its application for controlled roof caving in shallow depth seams," International Journal of Rock Mechanics and Mining Sciences, vol. 64, pp. 112-121, 2013.

[16] W. Chen, H. Ma, Z. Shen, and D. Wang, "Experiment research on the rock blasting effect with radial jet cracker," Tunnelling and Underground Space Technology, vol. 49, pp. 249-252, 2015.

[17] A. Markose and C. L. Rao, "Mechanical response of V shaped plates under blast loading," Thin-Walled Structures, vol. 115, pp. 12-20, 2017.

[18] H. Liang, P. Guo, K. Ye, B. Zhou, and Y. Yuan, "A study on crack propagation and stress wave propagation in different blasting modes of shaped energy blasting," Journal of Sound and Vibration, vol. 39, pp. 157-164, 2020.

[19] M. J. Coppinger, W. C. Uhlig, and J. H. J. Niederhaus, "Simulating lateral drift of a shaped charge jet in ALEGRA," International Journal of Impact Engineering, vol. 136, p. $103415,2020$.

[20] O. Ayisit, "The influence of asymmetries in shaped charge performance," International Journal of Impact Engineering, vol. 35, no. 12, pp. 1399-1404, 2008.

[21] M. Held, "Shaped charge optimization against ERA targets," Propellants, Explosives, Pyrotechnics, vol. 30, no. 3, pp. 216223, 2005.

[22] F. Zhu, Z. Liu, K. Gao, and J. Liu, "Experimental study on rock damage of roadway excavation by cumulative blasting in structural coal," Journal of Rock Mechanics and Geotechnical Engineering, vol. 37, pp. 2037-2047, 2018. 